26 Demers, G.W., Halbert, C.L. and Galloway, D.A. (1994) Virology 198, 169-174

27 Shadan, F.F., Cowsert, L.M. and Villareal, L.P. (1994) J. Virol. $68,4785-4796$

28 Cayrol, C. and Flemington, E.K. (1996) EMBO J. 15, 2748-2759

29 Wilcock, D. and Lane, D.P. (1991) Nature 349, 429-431

30 Jiang, D. et al. (1993) Oncogene 8, 2805-2812

31 Lechner, M.S. and Laimins, L.A. (1994) J. Virol. 7, 4262-4273

32 Yew, P.R., Liu, X. and Berk, A.J. (1994) Genes Dev. 8, 190-202

33 Subler, M.A., Martin, D.W. and Deb, S. (1992) J. Virol. 66 , 4757-4762

34 Von der Weth, A. and Deppert, W. (1993) J. Virol. 67, 886-893

35 Metz, T., Harris, A.W. and Adams, J. (1995) Cell 82, 29-36

36 Pan, H. and Griep, A.E. (1995) Genes Dev. 9, 2157-2169

37 Yanai, N. and Obinata, M. (1994) Exp. Cell Res. 211, 296-300

38 Han, J. et al. (1996) Genes Dev. 10, 461-477

39 Henderson, S. et al. (1991) Cell 65, 1107-1115

40 Allday, M.J.et al. (1995) EMBO J. 14, 1382-1391

41 Butz, K. et al. (1995) Oncogene 10, 927-936

42 Moore, M. et al. (1992) J. Virol. 66, 641-649

43 Scheffner, M. et al. (1991) Proc. Natl. Acad. Sci. U. S. A. 88, $5523-5527$

44 Hsu, I.C. et al. (1993) Carcinogenesis 14, 987-992

45 Crook, T. et al. (1992) Lancet 339, 1070-1073

46 Paquette, R.L. et al. (1993) Cancer 72, 1272-1280

47 Henkler, F. et al. (1995) Cancer Res. 55, 6084-6091

48 Unsal, H. et al. (1994) Proc. Natl. Acad. Sci. U. S. A. 91, 822-826
49 Rickinson, A.B. (1994) in Viruses and Cancer (Minson, A., Neil, J. and McCrae, M., eds), pp. 81-100, Cambridge University Press

50 Gerbes, A.L. and Caselmann, W.H. (1993) J. Hepatol. 19, 312-315

51 Gaidano, G. et al. (1991) Proc. Natl. Acad. Sci. U. S. A. 88, 5413-5417

52 Howard, J.C. et al. (1993) Oncogene 8, 2721-2729

53 Jacks, T. and Weinberg, R.A. (1996) Nature 180, 1-5

54 Ahmed, R., Morrison, L.A. and Knipe, D.M. (1996) in Fields Virology (Fields, B.N., Knipe, D.M. and Howley, P.M., eds), pp. 273-300, Lippincott-Raven

55 Brinster, R.L. et al. (1984) Cell 37, 367-379

56 Fukamizu, A. et al. (1994) J. Biol. Chem. 269, 31252-31258

57 Comerford, S.A. et al. (1995) Oncogene 10, 587-597

58 Kim, C.M. et al. (1991) Nature 351, 317-320

59 Faas, S.J. et al. (1987) J. Exp. Med. 165, 417-427

60 Apple, R.J. et al. (1994) Nat. Genet. 6, 157-162

61 Ellis, J.R.M. et al. (1995) Nat. Med. 1, 464-470

62 Midgley, C.A. et al. (1995) J. Cell Sci. 108, 1843-1848

63 Baxter, E.W. et al. (1996) J. Virol. 70, 2095-2100

64 Telerman, A. et al. (1993) Proc. Natl. Acad. Sci. U. S. A. 90, 8702-8706

65 Bischoff, J.R. et al. (1996) Science 274, 373-376

66 Slagle, B.L. et al. (1996) Mol. Carcinog. 151, 261-269

67 Huen, D.S. et al. (1993) J. Gen. Virol. 74, 1381-1391

68 Dequiedt, F. et al. (1995) Virology 209, 676-683

69 Haraf, D.J. et al. (1996) Clin. Cancer Res. 2, 755-762

\title{
Canine distemper virus - a morbillivirus in search of new hosts?
}

Timm C. Harder and Albert D.M.E. Osterhaus

\begin{abstract}
(
Tanine distemper virus (CDV) is a negativemorbillivirus (Paramyxoviridae) that infects a broad host spectrum among the Carnivora. This genus also includes measles virus (MV), rinderpest virus (RPV) and peste des petits ruminants virus (PPRV), which infect Artiodactyla. New mernbers of the genus, phocid distemper virus (PDV) and cetacean morbilliviruses, caused widespread severe epizootics among pinnipeds in 1988 in northwestern European waters and among toothed cetaceans in 1990-1991 in the Mediterranean, respectively ${ }^{1,2}$. The architecture and molecular
\end{abstract} biology of morbilliviruses are discussed in Box 1 .

Like all morbilliviruses, CDV is highly contagious, and transmission occurs predominantly via aerosols.
Canine distemper morbillivirus (CDV)

induces a multisystemic, often fatal disease

in a wide and seemingly expanding host range among the Carnivora. Several

genotypes of an otherwise monotypic virus species co-circulate in a geographically restricted pattern. Interspecies transmissions frequently occur, often leading to devastating epizootics in highly susceptible or immunologically naive populations.

T.C. Harder was at the Seal Rehabilitation and Research Centre, Pieterburen, The Netherlands and is now in the Institute of Medical Microbiology and Virology, Christian-Albrecht's University, Brunswiker Str. 4, D-24105 Kiel, Germany; A.D.M.E. Osterhaus * is in the Institute of Virology, Erasmus University, PO Box 1738 , 3000 DR Rotterdam, The Netherlands.

*tel: +3110408 8066, fax: +31104365145, e-mail: osterhaus@viro.fgg.eur.nl
In susceptible hosts, acute febrile and multisystemic disease is induced ${ }^{3}$; neuroinvasiveness and severe immunosuppression are hallmarks of CDV infection $^{4,5}$. Depending on the host species and the immune competence of the individual affected, mortality rates during CDV outbreaks can exceed $80 \%$.

It appears that since 1988 , an expansion of the alreadybroad natural host spectrum of CDV has occurred. Spontaneous, clinically overt infections with CDV-like morbilliviruses have been described in captive Japanese primates (Macaca fuscata $)^{6}$, collared peccaries (Tayassu tajacu ${ }^{7}$ and Lake Baikal seals (Phoca sibirica) ${ }^{8}$. Furthermore, distemper enzootics caused by CDV-like morbilliviruses have been observed for the first time in lions, tigers and jaguars kept in several North American 
zoos and safari parks ${ }^{9}$. In 1994, similar CDV-related disease outbreaks killed up to $30 \%$ of the free-ranging lion population and an unknown number of hyaenas in the Tanzanian Serengeti National Park and adjacent areas $^{10-14}$. Among the Felidae, domestic cats are known to be susceptible to experimental CDV infection, but evidence for natural infections, clinical disease or shedding of infectious virus has never been observed ${ }^{15}$. There is evidence for the involvement of CDV in Paget's disease of humans ${ }^{16}$. In addition to an apparently expanded host range of CDV, a resurgence of distemper among dog populations, in which high vaccination rates were maintained, has been observed since the late 1980 s, culminating in major epizootics in France, Germany and Scandinavia during 1991-1995 (Ref. 17).

Together, these observations have raised questions about the occurrence of variants of CDV that have allowed expansion into new hosts and challenge the suitability of current CDV vaccines.

\section{Biological and antigenic variants of CDV}

Efficacious modified CDV-live vaccines became available in the $1950 \mathrm{~s}$ and have been widely used since the 1960 s, leading to a drastically reduced impact of distemper on dog populations $^{18}$. Owing to the considerable degree of antigenic relationship between the different morbillivirus species, partial cross-protection against clinically overt CDV infections can also be induced in dogs following heterotypic vaccination with $\mathrm{MV}$ (Refs 19,20). Current licensed CDV vaccines are based on strains that have been attenuated by serial passage, either on canine kidney cells (Rockborn strain) ${ }^{21}$, hen eggs (original Onderstepoort strain) or chicken fibroblast cultures (Lederle strain) ${ }^{22}$.

Rockborn strain-based vaccines were shown to induce a higher titre of virus-neutralizing antibodies compared with Onderstepoort derivates and, consequently, avianized CDV vaccines are reported to cause higher vaccine failure rates ${ }^{23}$. The Rockborn strain, however, retains a certain pathogenic potential, particularly for wild species ${ }^{23}$. Although generally safe for use in healthy and immunocompetent dogs, both vaccine types remain extremely virulent for several carnivorous wild species such as red pandas (Ailurus fulgens), blackfooted ferrets (Mustela nigripes) or African wild dogs genome.

$3^{\prime}$

\section{Box 1. Molecular biology of morbilliviruses}

Morbillivirions consist of a single-stranded RNA genome of negative polarity ( $15900 \mathrm{bp}$ ), which is enclosed in a rod-shaped helical nucleocapsid (NC). Six transcription units, from which monocistronic mRNAs are generated, are ordered consecutively along the

\section{mRNA cascade}

\section{Negative-stranded genomic RNA}

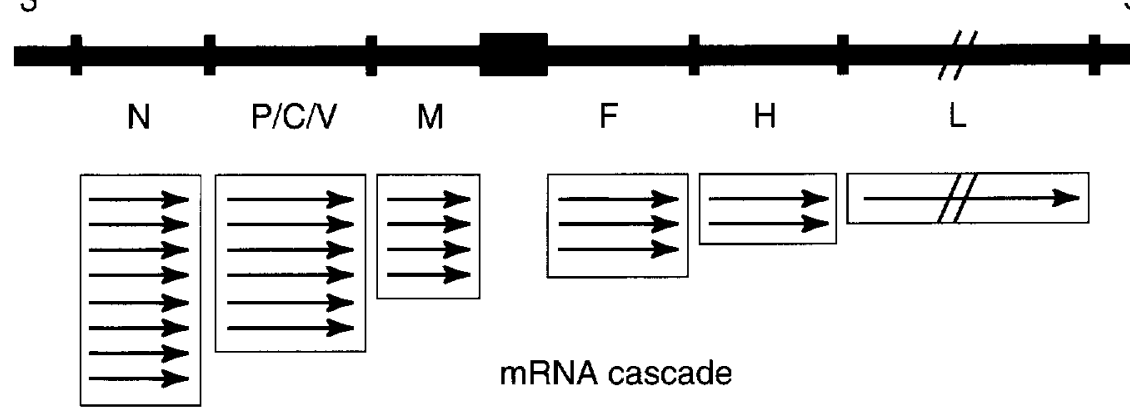

The entry site of the virion polymerase appears to reside in the $3^{\prime}$ leader sequence. At each intergenic region (red boxes) disintegration of the polymerase-RNA complex occur. Consequently, a steep gradient of mRNAs from the $\mathrm{N}$ gene (most abundant) to the $L$ gene (least abundant) is created. Exceptionally, the $P$ gene also encodes two Translation and processing

Regions within the morbillivirus genome that have been useful in the analysis of straindependent variation in measles virus (MV) and other morbilliviruses are shown as boxes. The long intercistronic region between the $M$ and $F$ genes (red box above) shows a high degree of plasticity between strains but is non-coding and, because of of the P gene (green box), which can be PCR-amplified from any morbillivirus species so-called 'universal morbillivirus primers', has been shown to be highly informa tive for phylogenetic and taxonomic purposes ${ }^{41}$. The NC is mainly formed by the $N$ protein but also contains copies of the polymerase-associated protein $(P)$ and the multifunctional RNA-dependent RNA virion polymerase ( $L$ ). The NC is enveloped by a host cell-derived lipid layer in which the viral attachment $(\mathrm{H})$ and fusion $(\mathrm{F})$ glycoproteins are embedded. In addition to glycosylation, the $\mathrm{F}$ protein undergoes endoproteolytic cleavage into two disulfide-linked fragments $\left(F_{1}-F_{2}\right)$, which is critical for functional activation. The blue boxes mark stretches of hydrophobic amino acids capable of spanning membranes. An internal layer of the matrix protein $(M)$ stabilizes the membrane and mediates contact with the NC.
(Lycaon pictus), emphasizing the species-related differences in susceptibility to CDV (Ref. 23). Although virulent and attenuated CDV biotypes can be distinguished by their ability to replicate in various macrophage, lymphocyte and epithelial cell cultures, other in vitro markers that differentiate unambiguously between these biotypes have not been defined. Vaccine strains replicate in all these cell types whereas virulent isolates require adaptation (often several blind passages) to replicate efficiently in epithelial cell lines such as Vero cells ${ }^{24,25}$. Among virulent CDVs a certain 


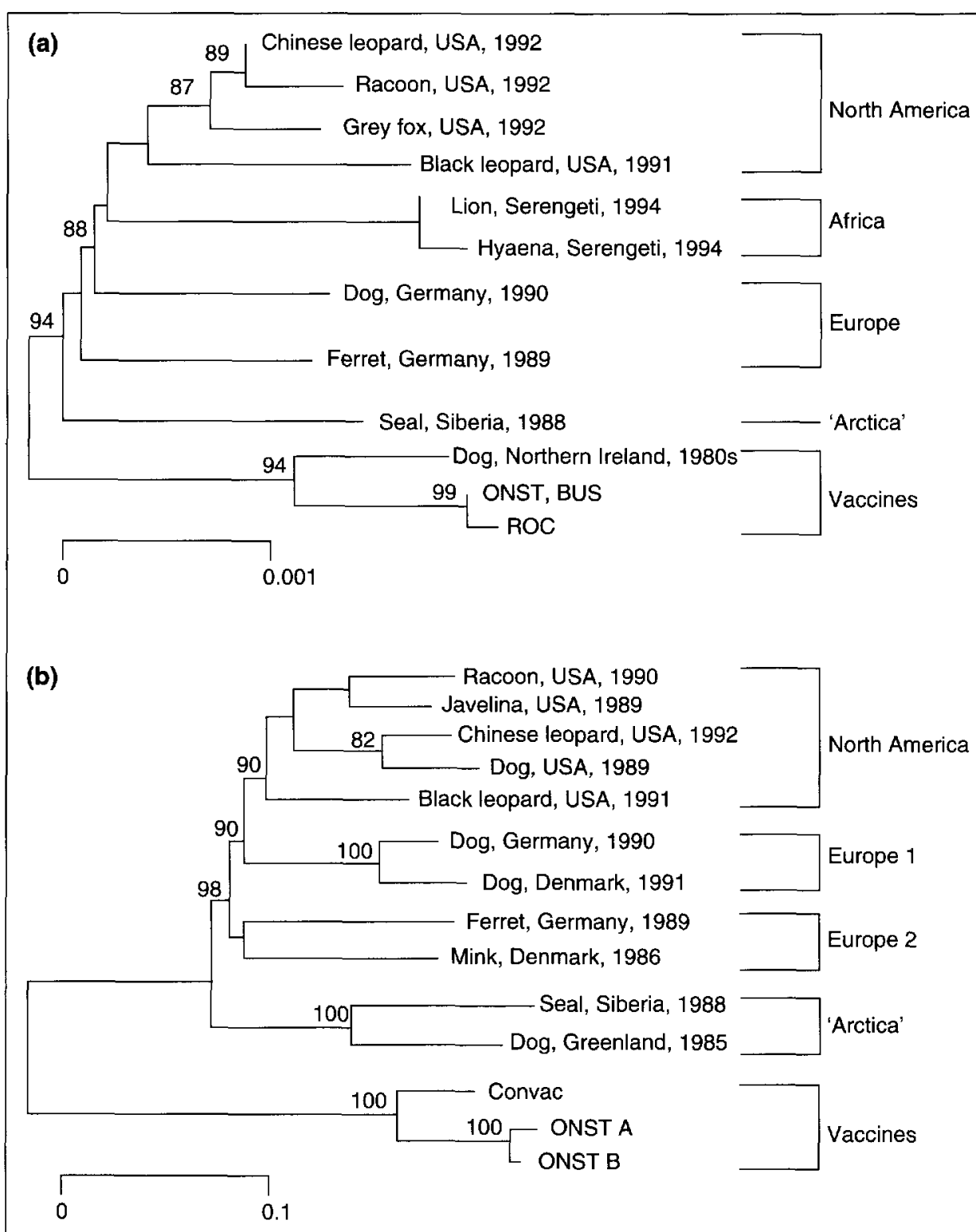

Fig. 1. Phylogenetic relationships between canine distemper virus (CDV) isolates are based on (a) analysis of a 388-bp fragment of the $P$ gene and (b) on the entire coding region of the $H$ gene (1821 bp). Genetic distances (see bars for scale), calculated by Kimura's 2-parameter method, have been used for tree construction employing the neighbour-joining principle. Identical topologies were obtained when sequences were analysed by maximum parsimony (not shown). Bootstrap values after 500 replications are given at tree branchings only when they exceed $80 \%$. Statistically significant cluster separation is assumed for values $>95 \%$. Computations were carried out using the $M^{M E G A}{ }^{R}$ PC software ${ }^{42}$. Sequences were extracted from the GenBank database and recent publica tions ${ }^{11-13,39,43}$. Host species, origin and year of isolation are indicated. Abbreviations: BUS, Bussell; ONST, Onderstepoort; ROC, Rockborn vaccine strains.

less, vaccine and wild-type CDV can be differentiated in crossneutralization and kinetic-neutralization assays $s^{9,13,28}$. Sera raised against wild-type CDV isolates have neutralization titres against the homologous virus that are up to tenfold greater than against vaccine strains of CDV (Ref. 13). Comparable serological results have been obtained for current MV wild-type isolates and vaccine strains $^{29}$. It is the membrane glycoprotein $\mathrm{H}$, the viral attachment factor, that induces the majority of neutralizing antibodies. Consequently, the observed differences in cross-neutralization titres should relate to antigenic variation in the $\mathrm{H}$ protein. However, it is difficult to differentiate between strains using monoclonal antibodies (mAbs) specific for the CDV $\mathrm{H}$ protein. So far, only one epitope has been identified on the $\mathrm{H}$ protein that is consistently expressed by CDV vaccine strains but not by most of the recent CDV wildtype isolates ${ }^{9,13,28,30,31}$. Variation has been observed in the apparent molecular weight of the CDV $\mathrm{H}$ protein: the $\mathrm{H}$ protein of the Onderstepoort strain migrates significantly faster than those of the Convac and Rockborn vaccine strains $^{32}$ or of various field isolates ${ }^{13}$. The lack of a demonstration of more-significant antigenic heterogeneity amongst CDV strains (including of other viral proteins such as N, P and F) may, at least in part, relate to the fact that all the CDV-specific mAbs used in these studies were raised against vaccine strains ${ }^{33}$.

\section{Molecular epidemiology of CDV}

Genetic analysis of a 388-bp P gene fragment and of the entire $\mathrm{H}$-protein-encoding region shows

degree of neuroinvasiveness appears to be straindependent and has been reported to correlate with plaque morphology in epithelial cell cultures ${ }^{26,27}$. The molecular basis of morbillivirus virulence is unknown. In the case of CDV and RPV infections at least, as yet undefined host species-specific, and possibly even breedspecific (in RPV), factors also play an important role ${ }^{3}$.

Serologically, all morbillivirus species are considered monotypic and the classical methods, including, for example, complement-fixation and immunofluorescence staining with polyclonal antibodies, do not distinguish serotypes among CDV strains. Neverthe- that several genotypes of wild-type CDV circulate in a geographically restricted pattern (Fig. 1; Box 2). In Europe, at least three different lineages co-circulated between 1989 and 1992. The CDV isolated from big cats of North American or African origin does not constitute a separate 'felid' distemper virus lineage but resembles the CDV circulating in local feral non-felid carnivores. Therefore, the big cat epizootics are likely to be caused by cross-species transmission of CDV indigenous to local wildlife or domestic carnivores ${ }^{13}$. It remains an enigma why CDV-related epizootics in these species have not occurred earlier or have not 
been noticed. Possibly, aggravating copathogens or predisposing genetic factors in these particular populations may have played a role and definitely require closer investigation ${ }^{14}$.

Within a group of four CDV isolates from European sources, those of canine origin can be distinguished from those in mustelids ranging in the same area (Fig. 1). Differences between the 'canine' and the 'mustelid' lineage are on average $3.4 \%$ and $4.2 \%$ at the nucleotide and the amino acid levels ( $\mathrm{H}$ gene and protein, respectively). This substantiates the assumption that these lineages have been co-circulating separately in this area for some time. However, further isolates of canine or mustelid origin from this area have not yet been analysed and interspecies transmissions between these lineages can not be excluded. Therefore, any conclusions about the existence of CDV lineages with host species (family) restriction in this area, which would also imply that the observed differences reflect a certain advantage to replicating in either mustelid or canine hosts, would be rash.

Another intriguing finding is the clustering of an isolate from a distemper-diseased dog (from Belfast, Northern Ireland) with vaccine CDV. Provided that laboratory contamination can be excluded, this case suggests that the comparatively old lineage from which all the vaccine strains have been derived has survived in the field for more than 40 years.

\section{Conclusions}

Considering the scarcity of data, the molecular epidemiology of CDV appears to be similar to that of RPV (Ref. 34) in that genetic variants form geographical clusters of serologically monotypic viruses. In $\mathrm{MV}$, up to eight genotypes have been distinguished so far, of which at least four appear to co-circulate globally $^{35,36}$. Genetic drift with a linear accumulation of mutations has been demonstrated for the N, P, M, F and $\mathrm{H}$ genes of $\mathrm{MV}$ (Refs $35,37,38$ ) and also for the $\mathrm{H}$ gene of currently circulating wild-type CDV. All outbreaks of morbillivirus-related disease in species previously not listed as natural hosts of CDV are caused by strains deeply rooted in the CDV cluster, favouring a scenario of interspecies transmission of CDV from local feral carnivores to large felids in the USA and in East Africa.

Antigenic drift in currently circulating wild-type CDV should be considered as a possible factor leading to a resurgence of distemper cases in well-vaccinated dog populations in Europe. The role of additional putative $\mathrm{N}$-glycosylation sites predicted in the $\mathrm{H}$ proteins of modern wild-type CDV needs to be elucidated ${ }^{13,39}$. Serological evidence indicates that the predicted amino acid changes accumulated in the $\mathrm{H}$ protein of circulating wild-type CDV have antigenic and probably immunological implications ${ }^{13}$, similar to the MV situation $^{29}$. In light of the fact that even heterotypic MV vaccination induces at least partial protection against challenge with virulent CDV in dogs ${ }^{19,20,40}$, it seems unlikely that modern virulent CDV strains are capable of breaking through a solid immunity mounted after vaccination with either of the common CDV vaccine

\section{Box 2. Phylogenetic analysis of CDV strains}

When sequences of the respective $P$ gene fragment of canine distemper virus (CDV) strains are used in phylogenetic analysis, a clustering of isolates becomes evident, which reflects geographic origin rather than host origin (Fig. 1a). A more-refined picture of the phylogenetic relationships among CDV strains is obtained when sequences of the gene encoding the $\mathrm{H}$ protein $(1821 \mathrm{bp})$ are analysed (Fig. 1b). The clusters obtained from phylogenetic analysis of the $\mathrm{H}$ gene and the $\mathrm{P}$ gene fragment are very similar, which renders recombination events between the lineages unlikely. At least four to five separate clusters of wild-type CDV can be distinguished, which differ by more than $0.5 \%$ at the nucleotide level ( $\mathrm{H}$ gene). According to the definition used in the analysis of measles virus (MV) strain variation ${ }^{44}$, these clusters are referred to as genotypes. The vaccine strains form a separate distinct lineage. At the amino acid level the greatest difference is observed between the $\mathrm{H}$ proteins of the Onderstepoort vaccine strain and a wild-type isolate obtained from a Chinese leopard (10.2\%). Wild-type CDV isolates express up to four additional putative $\mathrm{N}$-glycosylation sites located in the extracytoplasmic domain of the $\mathrm{H}$ protein when compared with the Onderstepoort vaccine strain ${ }^{13.39}$.

Even after prolonged serial passage in vitro, attenuated strains of MV and rinderpest virus (RPV) accumulate surprisingly few mutations within the coding regions compared with their virulent parents ${ }^{35,44,45}$. Consequently, it can be assumed that the differences between CDV vaccine strains (which originate from wild-type isolates made in the 1940 s and 1950s) and modern wild-type CDV are truly strain-dependent and do not reflect virulence variations.

strains. Nevertheless, epidemiologically, the situation may appear different when facing dog populations with critical CDV-specific herd immunity resulting from low vaccination rates or possibly from the frequent use of avianized vaccine derivates, which have been reported to be less efficient in inducing immunity compared with canine cell-culture-adapted vaccine strains ${ }^{23}$. Maintenance of high vaccination rates using efficacious vaccines that induce a solid, resilient immunity must still be given the highest priority in control of distemper, particularly in areas with high densities of dogs and their possible exposure to feral carnivores.

Recent morbillivirus epizootics have demonstrated that small populations of highly endangered species may be at risk of extinction when affected. However, efforts aimed at the protection of such populations, such as the African wild dog (L. pictus), by vaccination with proven safe, non-replicating vaccines need to be weighted meticulously against the negative impacts resulting from trapping and immobilizing free-ranging wild species.

Enlarging the collection of CDV-specific sequences obtained globally from a growing number of host species would provide the basis not only for understanding

\section{Questions for future research}

-Where are the reservoirs of virulent canine distemper virus (CDV)?

- Is perpetuation of CDV accomplished in low-density multispecies populations?

- Is there an antigenic drift in CDV?

- Do commercial CDV vaccine strains protect fully against currently circulating wild-type CDV?

- What is the molecular basis of virulence in CDV? 
the molecular epidemiology of CDV but also for the improvement of current CDV vaccines. In sharp contrast to MV, where humans are the only relevant reservoir $^{38}$, the broad natural host spectrum and the high likelihood of interspecies transmission of CDV make eradication impossible.

\section{Acknowledgements}

Work on morbillivirus molecular epidemiology by A.D.M.E.O. was supported by a HCM grant from the European Union (ERBCHBGCT920106). We acknowledge all colleagues who provided tissue samples or CDV isolates (particularly M. Appel and H. Lutz) and all coworkers in molecular analysis (particularly H. Vos and M. Kenter). We thank T. Barrett and L. Haas for supplying some sequences in computerreadable form before publication in GenBank and C. Kruyssen for assistance in preparing the manuscript.

\section{References}

1 Osterhaus, A.D.M.E. et al. (1989) Nature 335, 403-404

2 Visser, I.K.G. et al. (1993) J. Gen. Virol. 74, 631-641

3 Krakowka, S., Axthelm, M.K. and Johnson, G.C. (1985) in

Comparative Pathobiology of Viral Diseases (Vol. 2)

(Olson, R.G., Krakowka, S. and Blakeslee, J.R., eds), pp. 137-164, CRC Press

4 Krakowka, S. et al. (1980) Am. J. Vet. Res. 41, 284-292

5 Vandevelde, M. and Zurbriggen, A. (1995) Vet. Microbiol. 44, 271-280

6 Yoshikawa, Y. et al. (1989) Vet. Microbiol. 20, 193-205

7 Appel, M.J.G. et al. (1991) Arch. Virol. 119, 147-152

8 Grachev, M.A. et al. (1989) Nature 338, 209

9 Appel, M.J.G. et al. (1994) J. Vet. Diagn. Invest. 6, 277-288

10 Alexander, K.A. et al. (1995) J. Zoo Wildl. Med. 26, 201-206

11 Harder, T.C. et al. (1995) Vaccine 13, 521-523

12 Haas, L. et al. (1996) Vet. Microbiol. 49, 147-152

13 Harder, T.C. et al. (1996) J. Gen. Virol. 77, 397-405
14 Roelke-Parker, M.E. et al. (1996) Nature 379, 441-445 15 Appel, M.J.G. et al. (1974) Am. J. Vet. Res. 34, 1459-1463 16 Gordon, M.T. et al. (1992) Bone Miner. 19, 159-174

17 Blixenkrone-Möller, M. et al.,(1993) Vet. Microbiol. 37, 163-173

18 Gorham, J.R. (1966) J. Am. Vet. Med. Assoc. 149, 610-618

19 Baker, J.A. (1970) J. Am. Vet. Med. Assoc. 156, 1743-1746

20 Chalmers, W.S.K. and Baxendale, W. (1994) Vet. Rec. 135, 349-353

21 Rockborn, G. (1959) Nature 184, 822

22 Haig, D.A. (1956) Onderstepoort Vet. J. 27, 19-53

23 Appel, M.J.G. and Summers, B.A. (1995) Vet. Microbiol. 44, 187-191

24 Evans, M.B. et al. (1991) J. Vet. Diagn. Invest. 3, 127-132

25 Appel, M.J.G. et al. (1992) J. Vet. Diagn. Invest. 4, 258-263

26 Summers, B.A. et al. (1984) J. Comp. Pathol. 94, 65-75

27 Hirayama, N. et al. (1986) Jpn. J. Vet. Sci. 48, 259-265

28 Harder, T.C. et al. (1993) J. Virol. Methods 41, 77-92

29 Tamin, A. et al. (1994) J. Infect. Dis. 170, 795-801

30 Blixenkrone-Möller, M. et al. (1992) Arch. Virol. 123, 279-294

31 Alldinger, S. et al. (1993) Arch. Virol. 132, 421-428

32 Örvell, C. (1980) Arch. Virol. 66, 193-206

33 Örvell, C. et al. (1985) J. Gen. Virol. 66, 443-456

34 Chamberlain, R.W. et al. (1993) J. Gen. Virol. 74, 2775-2780

35 Rima, B.K. et al. (1995) in Measles Virus (Current Topics in Microbiology and Immunology) (Vol. 191), pp. 65-83, Springer-Verlag

36 Rima, B.K. et al. (1995) J. Gen. Virol. 76, 1173-1180

37 Rota, J.S. et al. (1992) Virology 188, 135-142

38 Rota, P.A. et al. (1995) Semin. Virol. 6, 379-386

39 Mamaev, L.V. et al. (1995) Vet. Microbiol. 44, 251-259

40 Appel, M.J.G. and Gillespie, J.H. (1972) Virol. Monogr. 11, 1-96

41 Barrett, T. et al. (1993) Virology 193, 1010-1012

42 Kumar, S. et al. (1994) CABIOS 10, 189-191

43 Mamaev, L.V. et al. (1996) Vet. Rec. 138, 437-439

44 Rota, J.S. et al. (1996) J. Infect. Dis. 173, 32-37

45 Baron, M.D. and Barrett, T. (1995) Vet. Microbiol. 44, 175-186

\section{AIDS policy in the UK}

\author{
AIDS in the UK. The Making of \\ Policy, 1981-1994 \\ by V. Berridge \\ Oxford University Press, 1996. \\ $£ 45.00 \mathrm{hbk}$ (xiv +389 pages) \\ ISBN 0198204728
}

$\mathrm{B}$ $y$ the end of 1995 , a cumulative total of 11872 cases of AIDS had been reported in the UK, the ninth highest rate of AIDS among the 44 countries reporting to the European Centre for Epidemiological Monitoring of AIDS. By comparison, incidence rates in France and Spain were more than 3 and 6 times higher, respectively. In retrospect, now that we know that a major heterosexual epidemic has not occurred in the UK, we can question whether AIDS has been handled appropriately or not. AIDS in the UK. The Making of Policy,
1981-1994 by Virginia Berridge, a historian at the London School of Hygiene and Tropical Medicine, is a must for anyone who is interested in this question.

Berridge divides her period of study (1981-1994) into four parts. The early years, from 1981-1985 ('Policy from below'), were ones of intensely hard work on the part of selected individuals in the absence of defined policy. It was during these years, however, that the defence of individual rights emerged as an issue and the groundwork was completed for the liberal official response. The years 1986 and 1987 ('The wartime response') saw intense official efforts to react to the epidemic, driven by a sense of emergency. Subsequently (1987-1989; Normalization and chronic disease: the power of epidemiology'), AIDS became viewed more as another health problem and less as a national threat. The final part of the book covers the period 1990-1994 ('The repoliticization of AIDS') and deals with a variety of issues, including that of HIV/AIDS in Africans in the UK, the recent efforts on the part of the gay community to emphasize that AIDS primarily affects gay men, and the campaign by a fringe minority to reject HIV as the cause of AIDS.

The strengths of this book are its prodigious detail and exhaustive background; Berridge has clearly done her homework and has talked to enormous numbers of people involved with HIV/AIDS. Although the comment may be unfair because the book is solely about the UK, I found it somewhat isolationist because the situation here forms such a small part of the overall world epidemic. Similarly, the fact that $10 \%$ of AIDS cases in the UK occur in Black Africans gets little attention, 